\title{
Recent Gamma-ray Results from DAMPE
}

\author{
Xiang Li ${ }^{* a}$, Kaikai Duan ${ }^{a b}$, Wei Jiang ${ }^{a c}$, Zhaoqiang Shen ${ }^{a}$ and Maria Muñoz Salinas ${ }^{d}$ \\ on behalf of the DAMPE collaboration ${ }^{\dagger}$ \\ ${ }^{a}$ Key Laboratory of Dark Matter and Space Astronomy, Purple Mountain Observatory, Chinese \\ Academy of Sciences, Nanjing 210034, China \\ ${ }^{b}$ University of Chinese Academy of Sciences, Beijing 100012, China \\ ${ }^{c}$ School of Astronomy and Space Science, University of Science and Technology of China, Hefei \\ 230026, China \\ ${ }^{d}$ Department of Nuclear and Particle Physics, University of Geneva, CH-1211 Geneva, \\ Switzerland \\ E-mail: खianqliepmo.ac.cn, duankkdpmo.ac.cn, Giangweidpmo.ac.cn, \\ zashendpmo.ac.cn, maria.munozdunige.ch
}

The Dark Matter Particle Explorer (DAMPE) is a space-borne high-energy charged cosmic ray and gamma-ray detector launched on December 17, 2015. After more than three years of operation in space, about 6 billion events are recorded, including 0.2 million photons above GeV energies. In this talk, we introduce some fundamental works of DAMPE. The preliminary results for the bright source list, the monochromatic gamma-ray line search and the pulsar analysis are also presented.

36th International Cosmic Ray Conference -ICRC2019-

July 24th - August 1st, 2019

Madison, WI, U.S.A.

\footnotetext{
${ }^{*}$ Speaker.

${ }^{\dagger}$ for collaboration list see PoS(ICRC2019)1177
} 


\section{Introduction}

The DArk Matter Particle Explorer (DAMPE) is a space-borne high energy particle detector dedicated to measure charged cosmic rays and gamma-rays in a broad energy range [प, [1] for the study of high energy astrophysics as well as the nature of dark matter particles [3], [, []]. It consists of a Plastic Scintillator strip Detector (PSD), a Silicon-Tungsten tracKer-converter (STK), a BGO imaging calorimeter (BGO) and a NeUtron Detector (NUD) (Fig. W).

DAMPE helps the study of gamma-ray detection, especially for its great potential in searching for the monochromatic and/or sharp spectral structures in $\mathrm{GeV}-\mathrm{TeV}$ range thanks to its excellent energy resolution [ [ $[$, 目]. In the following content, we first introduce some fundamental works for the gamma-ray analysis, which is followed by bright source list, gamma-ray line search and pulsar analysis.

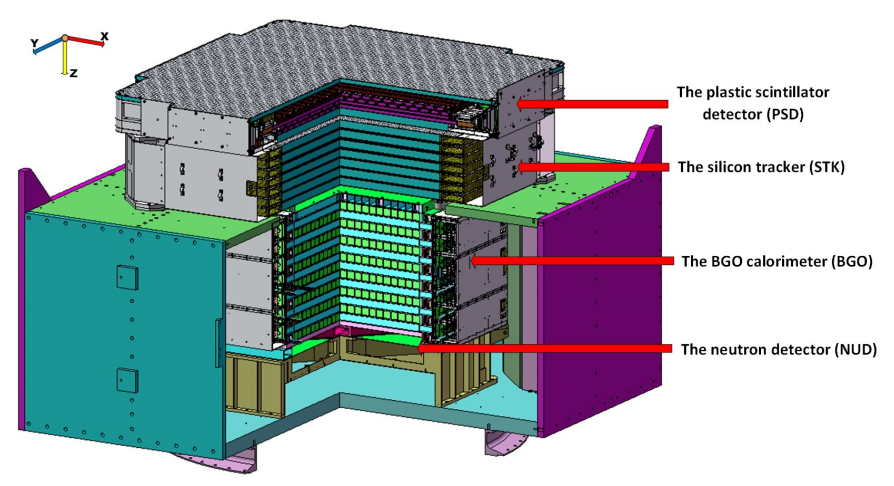

Figure 1: Schematic view of the DAMPE detector [వ]].

\section{Fundamental works: calibration, selection and IRFs}

DAMPE is operating in a $500 \mathrm{~km}$ solar-synchronous orbit with an inclination of $\sim 97^{\circ}$ and a period of about 95 minutes. During the on-orbit operation, we have been carrying out its calibrations since its launch [ $\square]$. Apart from the calibration with each sub-detector, we also performed some overall calibrations dedicated for gamma-ray detection such as the bore-sight alignment

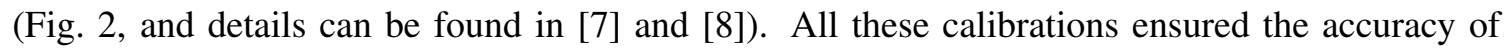
the data and also enhanced the precision.

Thanks to the powerful electromagnetic/hadronic shower discrimination provided by the calorimeter and the excellent charge rejection power provided by the PSD, we developed a sophisticated algorithm to resolve gamma-rays from the high charged cosmic ray background [Q]. Fig. [3] shows the counts map of the resolved gamma-rays in Galactic coordinate.

Instrument response functions (IRFs) are the parameterized representations of the instrument performance, and they are dependent on the algorithm of gamma-ray selection. The IRFs of DAMPE are factorized into three parts: the effective area, the point-spread function (PSF) and the energy dispersion function [ए0]. Fig. 目 shows an example of the IRFs of DAMPE with the use of a huge amount of Monte-Carlo data and the previous gamma-ray selection method. 


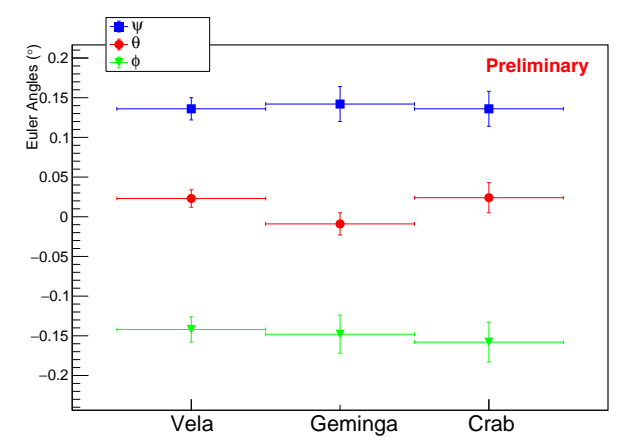

Figure 2: Bore-sight alignment results from three bright sources []$\left._{]}\right]$.

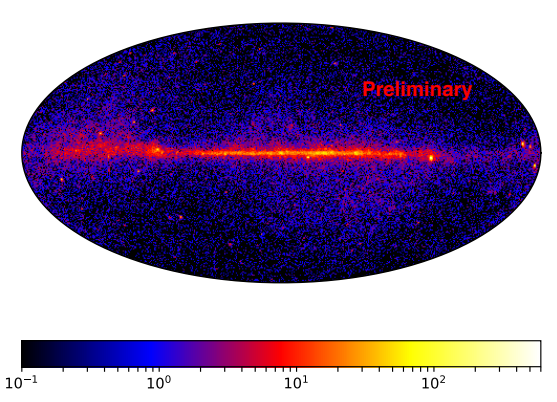

Figure 3: Gamma-ray counts map observed by DAMPE up to 31 Dec. 2018, with Aitoff projection in Galactic coordinate.

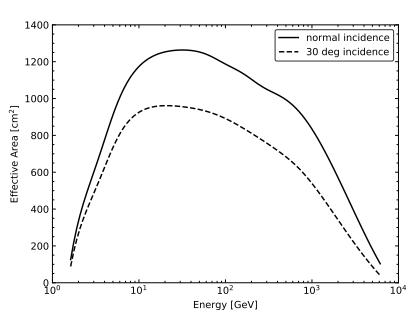

(a) Effective area [ [ $]$

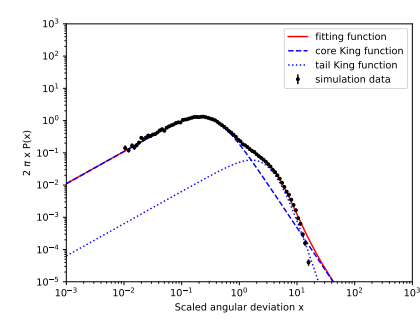

(b) Point-spread function [ए]]

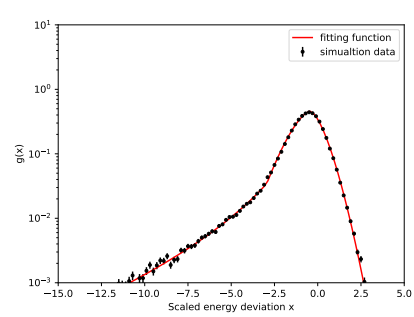

(c) Energy dispersion [0]]

Figure 4: An example of the IRFs of DAMPE.

\section{Bright Gamma-ray Source List}

In this section, we present the blind search method and results of DAMPE bright gamma-ray sources, and the sensitivity of DAMPE observation.

\subsection{Blind Search for Sources}

For searching sources observed by DAMPE, the data are selected with the energy range from $2 \mathrm{GeV}$ to $2 \mathrm{TeV}$ and time range up to $31 \mathrm{Dec} 2018$ for both Low-Energy-Trigger and High-Energy Trigger events. The data are further split into 15 logarithmically spaced energy bins.

We perform our blind search by calculating an all-sky TS map with the pixel size of around $0.1^{\circ}$. To speed up our analysis, we use $4^{\circ} \times 4^{\circ}$ region of interests (ROIs) which are the pixels of a HEALPix map [प]] with NSIDE=16. In each ROI, an Galactic diffuse emission model gll_iem_v06.fit from Fermi-LAT Collaboration [ए2] and an isotropic background with powerlaw spectrum are included in the gamma-ray model. Then we try to add a point-source with a power-law spectral type in each pixels of the ROI and test whether it is significantly shown. To do so, a binned analysis is performed with the spectral shape of point source and the normalization of Galactic and isotropic emissions free to fit. The test statistic (TS) value TS $\triangleq 2 \ln \left(L_{\text {with }} / L_{\text {without }}\right)$ is derived by optimizing the model with and without the assumed point source. 
From the all-sky TS map, we find 190 sources with TS value larger than 20, which are later used as seed sources. We then go through all the ROIs for one more time with all the seed sources within each ROI also included in the model. We fit the spectral parameters and positions of the sources and re-calculate the TS value. Finally, we get 143 and 120 sources with TS value larger than 20 and 25 respectively.

\subsection{Spectral Shape Analysis}

Only PowerLaw spectrum is used for blind search for sources, but the typical spectra of the AGNs and pulsars are curved or even with an exponential cutoff, and more detailed analyses for the spectra are needed.

For each detected sources, three spectral types are used to fit, including PowerLaw, LogParabola and PLSuperExpCutoff. ${ }^{1}$

The curved spectrum LogParabola and PLSuperExpCutoff are nested with PowerLaw. We define the confidence of curve $\mathrm{TS}_{\text {curve }} \triangleq 2 \ln \left(L_{\text {curvedSpectrum }} / L_{\text {PowerLaw }}\right)$. When $\mathrm{TS}_{\text {curve }}>9$, the source is considered significantly curved. If both the LogParabola and PLSuperExpCutoff are significant for a source, we choose the spectrum with the larger $\mathrm{TS}_{\text {curve }}$.

Following this method, 3 sources are considered with LogParabola spectrum and 2 sources with PLSuperExpCutoff. The flux of these sources are calculated with these spectrum.

\subsection{Association with 4FGL}

The fourth Fermi Large Area Telescope source catalog (4FGL) [[3] is based on the first eight years of science data from Fermi-LAT mission from $50 \mathrm{MeV}$ to $1 \mathrm{TeV}$. The 4FGL catalog includes 5098 sources above $4 \sigma$ significance.

We compare the position of sources detected by blind search with 4FGL, the distribution of the separation is $0.03 \pm 0.12$ degrees. All the blind search sources are included in the 4FGL, except for J1226.2+2151, which is a BL Lac object in 3FGL [प4]].

\subsection{Sensitivity}

For analysis the integral flux sensitivity of DAMPE gamma-ray observation, we put an assumed point source at the center of the pixels in HEALPix projection with NSIDE $=64$, along with the Galactic diffuse emission and isotropic emission, to compose the model of sky, here the effect of the point sources is ignored.

The point source is assumed with PowerLaw spectrum with a fixed index of 2 . We vary the flux the point source until the TS of the source up to 25, and the flux is considered as the sensitivity of DAMPE. Going through the whole sky, we get the integral flux sensitivity of DAMPE for gammaray observation Fig. [1.

The integral flux sensitivity is dependent on the spectral index, so we define the broadband sensitivity as the maximum flux of source at the DAMPE detection threshold for any spectral index $(0.5-3.5)$. In practice this is calculated from the envelope of power-law spectral models at the DAMPE detection threshold (the left panel of Fig. (6). The right panel of Fig. 6 shows the

\footnotetext{
${ }^{1}$ Descriptions for these spectral models can be found in https://fermi.gsfc.nasa.gov/ssc/data/ analysis/scitools/source_models.html.
} 
broadband source sensitivity evaluated for four different locations: Galactic center, intermediate latitudes, north Galactic pole, and north Celestial pole.

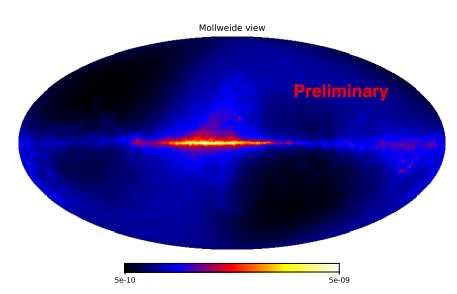

Figure 5: Integral flux sensitivity of DAMPE for gamma ray observation.
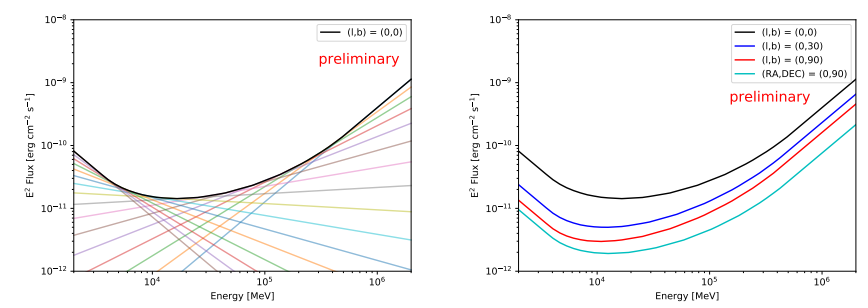

Figure 6: Broadband sensitivity of DAMPE observation for Galactic center, intermediate latitudes, north Galactic pole, and north Celestial pole.

\section{Gamma-ray line search}

Dark matter (DM) particles may annihilate to gamma-ray pairs and therefore create line-like signals which can be a smoking-gun signature. Thanks to its excellent gamma-ray energy resolution, DAMPE can be a powerful telescope for line detections [0]. We search for lines between 10 and $300 \mathrm{GeV}$ based on 3-year observations of the Galactic center (GC) and some nearby galaxy clusters (GCls) using the sliding windows technique with unbinned likelihood.

In the analyses of GC, we choose several optimized ROIs according to the DM distributions we assumed. For the cuspy DM profiles, we use small radii to constrain the astrophysical backgrounds; while for the flat profiles, larger ROIs are adopted to include more signal events. Furthermore, the Galactic plane region distant from the $\mathrm{GC}\left(|b|<5^{\circ}\right.$ and $|l|>6^{\circ}$, where $l, b$ stand for the longitude and latitude in Galactic coordinate) is excluded from the analyses. The ROI is denoted with $\mathrm{R} x$, which has a radius of $R=x^{\circ}$ centering at the GC excluding the Galactic plane. According to the sliding windows analyses, no line structure with the local significance larger than $3 \sigma$ is found. The left panel of Fig. $\square$ shows the $\mathrm{TS}_{\text {line }}$ [ㄷ]] with different energies derived with the ROI R90 (more results are given in [[15]). Since no line is found, we calculate the $95 \%$ upper limit of the DM annihilation cross sections to a pair of photons for each DM density distribution. The right panel of Fig. $\square$ shows the upper limit derived from the ROI R90, which is quite close to the 5.8-year result of Fermi-LAT for the same ROI in [ए]] thanks to the excellent energy resolution of DAMPE. However, as to the small ROIs, since Fermi-LAT has more exposure at GC, our constraints are still weak.

We also search for lines in the GCl samples from the HIFLUGCS catalog [1], [8] which contain 16 and 29 clusters with the largest $J$-factors respectively. Fig. $\mathbb{8}$ shows the results derived with the $16 \mathrm{GCl}$ sample made by [एव]. Clearly, no significant line structure is found.

Details can be found in [ㄷ]].

\section{Pulsars}

Pulsars are highly magnetized and rapidly rotating neutron stars. Neutron stars are extremely 

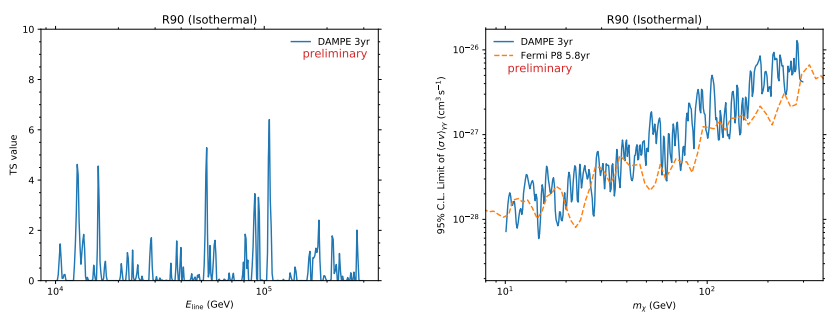

Figure 7: The TS values of a series of line energies in the sliding window analysis of the GC with $90^{\circ}$ ROI (left panel) and the 95\% confidence level upper limit on the DM annihilation cross section (right panel).

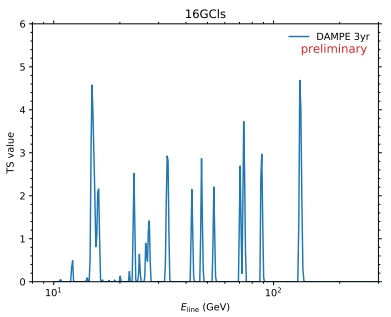

Figure 8: The TS values of a series of line energies in the sliding window analysis of the 16 GCls.

dense objects, that are constantly used as astrophysical laboratories that help to test the constantly struggling relation between astrophysics, nuclear physics and particle physics.

The different ways to detect a pulsar are dependent on the acceptance of the instrument and on its orbit. In the case of DAMPE we do a search based on known pulsars and then use models calculated by other experiments, normally in different wavelength such as radio, X-ray or even gamma rays.

We select events of interest for pulsars around their known positions. The barycenter correction is done for relativistic effects related to time dilatation. This time variations are very small but important and consider effects such as Jupiter's and the Sun's orbit. Finally we get the Barycentric Dynamical Time (TDB). The position of the known source also need to be precise, therefore proper motion i.e., motion of the pulsar is also considered. Then we fold the TDB times with the ephemeris to get the pulsar phases, by using the TEMPO2 software package [RO].

At the moment more than 20 pulsars have been studied with DAMPE data set, though only a small fraction of them have enough statistics. Fig. 8 shows the phases of Vela, Geminga, J17094429, the Crab and J0007+7303, whose significances are greater than five.

Details can be found in [2]].

\section{Summary}

DAMPE has been operating stably on orbit for more than three years and more than 6 billion charged cosmic ray and gamma-ray events covering a very wide energy range have been collected, within which about 0.2 million are gamma-rays. With the complete fundamental works, we are using the collected data to carried out some gamma-ray scientific works. Independent contributions related to DAMPE gamma-ray research are also presented elsewhere, including the line searching [ㄷ]], the pulsars [2]] and the bore-sight alignment [《]].

\section{Acknowledgements}

The DAMPE mission is funded by the strategic priority science and technology projects in space science of Chinese Academy of Sciences. In China the data analysis is supported in part by the National Key Research and Development Program of China (No. 2016YFA0400200), the 


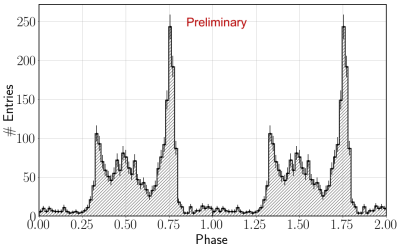

(a) Vela

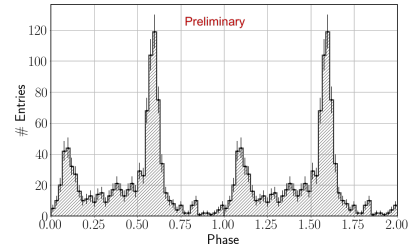

(b) Geminga

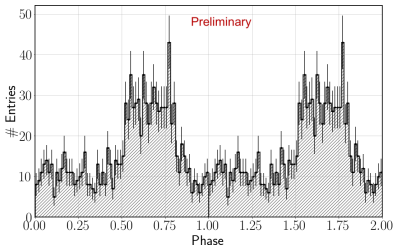

(c) J1709-4229

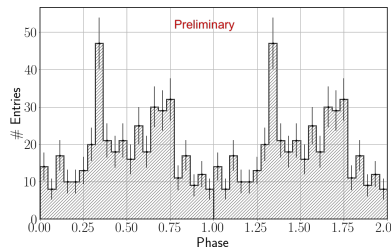

(d) $\mathrm{Crab}$

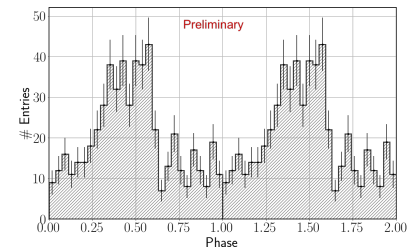

(e) J0007+7303

Figure 9: Results of the five pulsars above five sigmas.

National Natural Science Foundation of China (Nos. 11525313, 11622327, 11722328, U1738205, U1738207, U1738208, 11851305, U1738138), the strategic priority science and technology projects of Chinese Academy of Sciences (No. XDA15051100), the 100 Talents Program of Chinese Academy of Sciences, the 13th Five-year Informatization Plan of Chinese Academy of Sciences (XXH13506), and the Young Elite Scientists Sponsorship Program. In Europe the activities and the data analysis are supported by the Swiss National Science Foundation (SNSF), Switzerland; the National Institute for Nuclear Physics (INFN), Italy.

\section{References}

[1] J. Chang, Dark Matter Particle Explorer: The First Chinese Cosmic Ray and Hard $\gamma$-ray Detector in Space, Chin. J. Spac. Sci. 34 (2014) 550.

[2] J. Chang et al. (DAMPE Collaboration), The DArk Matter Particle Explorer mission, Astropart. Phys. 95 (2017) 6.

[3] Y.-Z. Fan, B. Zhang and J. Chang, Electron/positron excesses in the cosmic ray spectrum and possible interpretations, Int. J. Mod. Phys. D, 19 (2010) 2011.

[4] J. L. Feng, Dark matter candidates from particle physics and methods of detection, Annu. Rev. Astron. Astrophys., 48 (2010) 495.

[5] Y.-F. Liang, Z.-Q. Shen, X. Li et al., Search for a gamma-ray line feature from a group of nearby galaxy clusters with Fermi LAT Pass 8 data, Phys. Rev. D, 93 (2016) 103525.

[6] S.-J. Lei, Q. Yuan, Z.-L. Xu, K.-K. Duan, and M. Su, Gamma-ray Astronomy with DAMPE, in proceedings of ICRC2017, POS(ICRC2017) 616 (2017).

[7] G. Ambrosi et al. (DAMPE Collaboration), The on-orbit calibration of DArk Matter Particle Explorer, Astropart. Phys. 106 (2019) 18. 
[8] W. Jiang, X. Li, K.-K. Duan, J.-J. Zang and S.-J. Lei, Boresight alignment with the DArk Matter Particle Explorer, in proceedings of ICRC2019, POS (ICRC2019) 567 (2019).

[9] Z.-L. Xu, K.-K. Duan, Z.-Q. Shen, et al., An algorithm to resolve $\gamma$-rays from charged cosmic rays with DAMPE. Research in Astronomy and Astrophysics, 18 (2018) 027.

[10] Kai-Kai Duan, Wei Jiang, Yun-Feng Liang et al., DmpIRFs and DmpST: DAMPE Instrument Response Functions and Science Tools for Gamma-Ray Data Analysis, [arXiv: 1904.13098].

[11] K. M. Górski, E. Hivon, A. J. Banday et al., HEALPix: A Framework for High-Resolution Discretization and Fast Analysis of Data Distributed on the Sphere, Astrophys. J., 622 (2005) 2.

[12] F. Acero et al. (Fermi-LAT Collaboration), Development of the model of Galactic interstellar emission for standard point-source analysis of Fermi Large Area Telescope data, Astrophys. J Suppl. Ser. 223 (2016) 26.

[13] The Fermi-LAT collaboration, Fermi Large Area Telescope Fourth Source Catalog, [arXiv:1902.10045].

[14] F. Acero et al. (Fermi-LAT collaboration), Fermi Large Area Telescope Third Source Catalog, Astrophys. J Suppl. Ser. 218 (2015) 23.

[15] Z.-Q. Shen, Y.-F. Liang, K.-K. Duan, X. Li, and Y.-Z. Fan, Search for a gamma-ray line feature with DAMPE, in proceedings of ICRC2019, POS (ICRC2019) 601 (2019).

[16] M. Ackermann et al. (Fermi-LAT Collaboration), Updated search for spectral lines from Galactic dark matter interactions with pass 8 data from the Fermi Large Area Telescope, Phys. Rev. D 91 (2015) 122002.

[17] T. H. Reiprich and H. Böhringer, The Mass Function of an X-Ray Flux-limited Sample of Galaxy Clusters, Astrophys. J. 567 (2002) 716.

[18] Y. Chen, T. H. Reiprich, H. Böhringer, Y. Ikebe, and Y.-Y. Zhang, Statistics of X-ray observables for the cooling-core and non-cooling core galaxy clusters, Astron. Astrophys., 466 (2007) 805.

[19] B. Anderson, S. Zimmer, J. Conrad, M. Gustafsson, M. Sánchez-Conde, and R. Caputo, Search for gamma-ray lines towards galaxy clusters with the Fermi-LAT, J. Cosmol. Astropart. Phys. 02 (2016) 026.

[20] G. B. Hobbs, R. T. Edwards, and R. N. Manchester, TEMPO2, a new pulsar-timing package - I. An overview, MNRAS, 369 (2006) 655.

[21] M. Muñoz, X. Wu, F. Gargano, K.-K. Duan and Z.-Q. Shen, Gamma-ray pulsars with DAMPE, in proceedings of ICRC2019, POS (ICRC2019) 587 (2019). 